07

\title{
Закономерности микродеформации сверхвысокомолекулярного полиэтилена, модифицированного добавками галлуазита
}

\author{
(C) В.В. Шпейзман ${ }^{1}$, П.Н. Якушев ${ }^{1}$, В.М. Егоров ${ }^{1}$, С.В. Васильева ${ }^{2}$, А.С. Смолянский ${ }^{2,3}$ \\ ${ }^{1}$ Физико-технический институт им. А.Ф. Иофрфе РАН, \\ Санкт-Петербург, Россия \\ ${ }^{2}$ Филиал АО „Ордена Трудового Красного Знамени научно-исследовательский \\ физико-химический институт им. Л.Я. Карпова“, \\ Москва, Россия \\ ${ }^{3}$ Российский химико-технологический университет им. Д.И. Менделеева, \\ Москва, Россия \\ E-mail: shpeizm.v@mail.ioffe.ru
}

(Поступила в Редакцию 16 ноября 2017 г.

В окончательной редакции 9 января 2018 г.)

\begin{abstract}
Исследовано влияние добавок 1 и 3 mass\% галлуазита на скорость и малые скачки деформации при одноосном сжатии сверхвысокомолекулярного полиэтилена. Прецизионная интерференционная методика измерения скорости с разрешением по перемещению $325 \mathrm{~nm}$ и по частоте $1 \mathrm{kHz}$ позволила обнаружить несколько уровней деформации в микро- и нанометровым диапазонах. Показано, что добавление галлуазита приводит к уменьшению скорости деформации при одинаковых условиях нагружения и изменению характеристик скачков деформации. Калориметрические измерения показали, что при плавлении полиэтилена с разным содержанием галлуазита происходит изменение энергии перехода и степени кристалличности.
\end{abstract}

Настоящее исследование проведено при поддержке Российского фонда фундаментальных исследований (проект № 17-07-00524).

DOI: $10.21883 /$ FTT.2018.07.46120.323

\section{1. Введение}

Интерес к природным трубчатым материалам, каким является галлуазит (Hal), связан с широкими возможностями его использования в различных областях техники. Благодаря хорошей биосовместимости, они могут найти применение также в медицине и биологии. В полимеры Hal добавляют для повышения прочности и модуля упругости, термостабильности и огнестойкости, предотвращения коррозии. Подробное описание структуры, биохимических и физических свойств как самого галлуазита, так и композитов, содержащих Hal, представлено в многочисленных статьях и обзорах (см., напримep, $[1-4])$.

Большинство исследований механических свойств композитов показало, что и модуль упругости, и прочность при растяжении возрастают с ростом содержания галлуазита. Так, для полиметилметакрилата прочность при растяжении увеличивается при добавлении 2 mass $\%$ Hal на $30 \%, 5$ mass $\%$ - в 2 раза и 10 mass \% - в 2.5 раза [4]. Модуль упругости композита полиамид (PA-6) - $30 \mathrm{mass} \%$ Hal в 2 раза выше, чем у чистого РА-6 [5]. Аналогичное изменение деформационно-прочностных характеристик наблюдалось при добавлении Hal в эпоксидные смолы, карбоксилированный бутадиен-стирольный каучук, поливинилацетат (PVA) и др. [1,3,6].

В отличие от перечисленных выше полимеров влияние галлуазита на механические свойства композита
полиэтилен-Hal оказалось неоднозначным. При исследовании полиэтилена высокой плотности (PEHD), содержащего 1-10 mass\% Hal, было показано [7], что прочность при растяжении практически не изменяется. При составе композита PEHD-10 mass\% Hal модуль упругости увеличивается на $10 \%$, а предельное удлинение уменьшается на $20 \%$. С ростом содержания галлуазита в составе композита прочность на изгиб и модуль упругости возрастают на $17 \%$ для модуля упругости и $12 \%$ для прочности при 5 mass\% Hal. Увеличение содержания Hal c 5 до 10 mass\% не показало значительного влияния на изгибные свойства. При этом довольно сильно (до 30\%) снизилась ударная вязкость. Также лишь небольшое улучшение механических свойств наблюдалось в композите полипропилен-Hal [8].

Как правило, в исследованиях [1-8] определяли предельные величины допустимых напряжений (прочности) и деформаций в полимерных композициях, содержащих значительные количества наполнителя; при этом для многих изученных композитов значения предельной деформации составляли десятки процентов. В то же время не рассматривался практически важный случай поведения полимерных композитов с добавкой галлуазита при малых деформациях и напряжениях. В настоящем исследовании с помощью прецизионной интерференционной методики определения скорости и величины деформации было исследовано влияние добавок Hal на скорость деформации при небольших напряжениях. 


\section{2. Образцы и методика исследования}

Исследовались образцы сверхвысокомолекулярного полиэтилена (UHMV PE) марки Hi-Zex Million, молекулярная масса $4.5 \cdot 10^{6}$ атомных единиц, средний размер частиц $150 \mu \mathrm{m}$, производства фирмы Mitsui PetroChemical Industries (Япония) и композита UHMV PE-галлаузит, содержащего 1 или 3 mass\% Hal.

Диски UHMV PE и композита диаметром 38.5 и высотой $10 \mathrm{~mm}$ получали посредством термопрессования порошка исходного UHMV PE и смесей порошков UHMV PE и Hal, взятых в приведенных выше весовых соотношениях. В процессе спекания сначала полуфабрикат должен прнять форму диска (стадия пластикации), затем уплотниться. Пластикацию проводили при $2-5 \mathrm{MPa}$ в течение $2-3 \mathrm{~h}$ при температурах от 150 до $170^{\circ} \mathrm{C}$. Время спекание заготовки составляло $2-3 \mathrm{~h}$, температура от 200 до $240^{\circ} \mathrm{C}$, величина приложенного давления от 8 до $10 \mathrm{MPa}$. По завершении стадии спекания убирали давление и охлаждали диски в пресс-форме до комнатной температуры. Экспериментальные образцы в виде цилиндров диаметром 3 и высотой $10 \mathrm{~mm}$ вырезали из центральной части дисков методом микрофрезерования, а затем торцы образцов шлифовали до достижения высоты $6.0 \pm 0.1 \mathrm{~mm}$.

В процессе деформационных испытаний образцы UHMV PE и композита UHMV PE-Hal подвергались одноосному сжатию. Сжатие проводилось на установке для измерения ползучести, в которой скорость деформации определялась с помощью лазерного интерферометра [9-12]. По частоте биений на интерферограмме определяли скорость, а по числу биений - величину деформации. Частота дискретизации при записи кривых деформации и ее скорости составляла 0.5 или $1 \mathrm{\kappa Hz}$, что намного меньше верхней границы полосы пропускания аппаратуры $(\sim 20 \mathrm{kHz})$. При вычислении $\varepsilon$ и $\Delta \varepsilon$ в расчет принималась полная длина образца.

Определение скорости деформации производилось на базе последовательных одинаковых изменений длины образца $\Delta l_{0}=325 \mathrm{~nm}\left(\Delta l_{0}=\lambda / 2\right.$, где $\lambda$ - длина волны лазера, используемого в установке), что соответствовало полному периоду биения. Специально разработанное программное обеспечение (ПО) позволяло производить сбор, обработку и хранение экспериментальной информации в режиме „on-line“ [13]. Также обработку экспериментальных данных проводили с применением ПО MATLAB и Origin 9.0.

Под „скачком“ деформации мы понимаем величину деформации между близкими по величине скачками скорости деформации, т.е. период следования этих скачков по шкале перемещений. Скачки деформации, превышающие $\sim 600 \mathrm{~nm}$, рассчитывали по зависимостям скорости относительной деформации $\varepsilon$ от деформации с шагом $\Delta l_{0}$ путем измерения периодов колебаний скорости $L$. Таких периодов может быть несколько. Как показали наши предыдущие исследования [12-14], существуют 2-3 характерных для выбранных условий деформации размера, около которых группируются все наблюдаемые скачки скорости деформации. При этом существует иерархия скачков, согласно которой более мелкие скачки входят в состав следующих по величине скачков. Для групп скачков близких размеров рассчитывалось среднеквадратичное отклонение скорости от ее величины на сглаженной зависимости скорости деформации от времени.

Скачки, меньшие $325 \mathrm{~nm}$, определялись по отклонению формы колебания на экспериментальной кривой биений от расчетной, описывающей интерференцию двух высокочастотных колебаний с близкими частотами [12]. При этом исходили из предположения, что отклонения определяются изменением частоты, т. е. скорости деформации. Величина скачка нормировалсь на деформацию одного биения, т.е. их суммарный вклад в деформацию составлял $0.325 \mathrm{~nm}$. Распределение скачков по размерам не было равномерным. Так же, как и для микрометровых скачков, определяли их наиболее вероятные рамеры $L_{1}$ и среднеквадратичное отклонение величины сигнала на экспериментальной кривой от расчетной. Скачки меньшего размера входили в состав более крупных скачков, а наибольшие нанометровые скачки образовывали микрометровые скачки наименьшего размера.

Скорость деформации $\varepsilon$ при постоянных напряжениях быстро затухала. Основные измерения проводились для скоростей в интервале $\dot{\varepsilon}=10^{-6}-5 \cdot 10^{-4} \mathrm{~s}^{-1}$. При выбранной частоте дискретизации записи (например, $1 \mathrm{kHz}$ ) и скорости деформации $\dot{\varepsilon}=10^{-5} \mathrm{~s}^{-1}$ (время одного биения $\sim 5 \mathrm{~s}$ ) одно полное колебание на интерферограмме оказывается оцифрованным $5 \cdot 10^{3}$ точками с интервалом между ними $1 \mathrm{~ms}$. Если считать, что для регистрации одного биения с целью дальнейшей компьютерной обработки сигнала достаточно, например 5 точек, то мы можем корректно измерять скорости в $10^{3}$ раз большие, чем средняя, т.е. $10^{-2} \mathrm{~s}^{-1}$. Однако реально наблюдаемые единичные большие скачки имели в десятки раз меньшую скорость. ${ }^{1}$

Собственную частоту системы образец-груз, необходимый для требуемого сжатия образца, можно приближенно оценить как частоту $f$ осцилляций груза массой $m=\sigma S / g$ на пружине жесткостью $C=S E / l$, где $\sigma, S$ и $l-$ соответственно, напряжение сжатия, площадь поперечного сечения и длина образца, $g$ ускорение свободного падения. Тогда для принятых в работе условий опытов $f=(2 \pi)^{-1}(E g / l \sigma)^{0.5} \approx 24 \mathrm{~Hz}$, а переходные времена в системе $t \approx(2 \pi f)^{-1} \approx 6 \mathrm{~ms}$. С этими величинами ниже будем сравнивать характерные размеры скачков разной величины.

Помимо измерений скорости и величины деформации, методом дифференциальной сканирующей калори-

\footnotetext{
1 Заметим, что максимальная частота, дискретизации записи в разработанной программе составляет $10^{4} \mathrm{~Hz}$ и, если потребуется, может быть увеличена. Однако при большой дискретизации записи проиходится исследовать очень малые перемещения, в противном случае файлы записи имеют очень большой объем, и с ними неудобно работать.
} 
метрии был измерен тепловой эффект при плавлении UHMV PE и композита UHMV PE-Hal с содержанием галлуазита 1 и 3 mass\%.

\section{3. Результаты и обсуждение}

На рис. 1, $a, b, c$ приведены зависимости скорости деформации $\varepsilon$ от времени $t$ при сжатии чистого UHMV PE (рис. $1, a)$ и композита UHMV PE-Hal с содержанием галлуазита 1 (рис. $1, b)$ и 3 mass\% (рис. $1, c$ ) при одном и том же напряжении $\sigma=15 \mathrm{MPa}$. Там же показаны кривые ,деформация-время“, в которых деформация определялась по числу биений от начала измерения. Как и для многих других материалов [9-13], скорость деформации, измеренная на малом перемещении с высокой частотой записи, содержит скачки различной величины, в то время как кривая деформации сглаживает эти скачки и выглядит плавной (см. вкладки на рис. 1). Чтобы получить величину скачков скорости деформации, т.е. построить базовую кривую, от которой отсчитывать величину скачка, было использовано усреднение с большой величиной окна $(\sim 160 \mu \mathrm{m})$ с шагом $325 \mathrm{~nm}$. Сглаженные зависимости скорости деформации от времени приведены на рис. $1, a-c$ в виде сплошных линий и отдельно на рис. 2.

Из сопоставления зависимостей $\dot{\varepsilon}-t$ для всех исследованных материалов было обнаружено, что в области использованных напряжений скорость деформации уменьшалась со временем. Роль добавки галлуазита проявляется в ускорении этого процесса, т. е. при одинаковом времени максимальная деформация наблюдается у исходного UHMV PE, минимальная - у композита UHMV PE - 3 mass\% Hal). Следовательно, введение 1-3 mass\% Hal в UHMV PE повышает сопротивление деформации полимера в области малых напряжений.

Также следует отметить высокую неравномерность процесса деформации, как в исходном UHMV PE, так и в композитах UHMV PE- $(1-3$ mass $\%$ Hal $)$. В качестве примера на рис. 3 приведен участок кривой биений, зарегистрированной при деформации композита UHMV PE-1 mass\% Hal, в котором соседние биения отличаются по частоте (скорости деформации) в 5 раз. В отдельных случаях, преимущественно на начальной стадии деформационного процесса, частота соседних биений могла отличаться в десятки раз.

Перестроив графики рис. 1 в координатах $\Delta \dot{\varepsilon} / \dot{\varepsilon}-(\Delta l)$, где $\Delta \dot{\varepsilon} / \dot{\varepsilon}-$ относительная разница между значениями скорости деформации на сглаженной кривой и поученными в эксперименте, а $\Delta l$ - текущее изменение длины образца, можно определить размеры скачков деформации $L$ и среднеквадратичные отклонения $S_{\overline{\Delta \dot{\varepsilon} / \varepsilon}}$ на разных уровнях деформации. На рис. 4 приведена подобная кривая для композита UHMV PE-3 mass\% Hal. Аналогичные зависимости были получены для исходного UHMV PE и композита UHMV PE-1 mass\% Hal.
По числу измеренных на кривых $\Delta \dot{\varepsilon} / \dot{\varepsilon}-\Delta l$ расстояний между скачками скорости деформации были построены кривые распределения микрометровых скачков по разме-
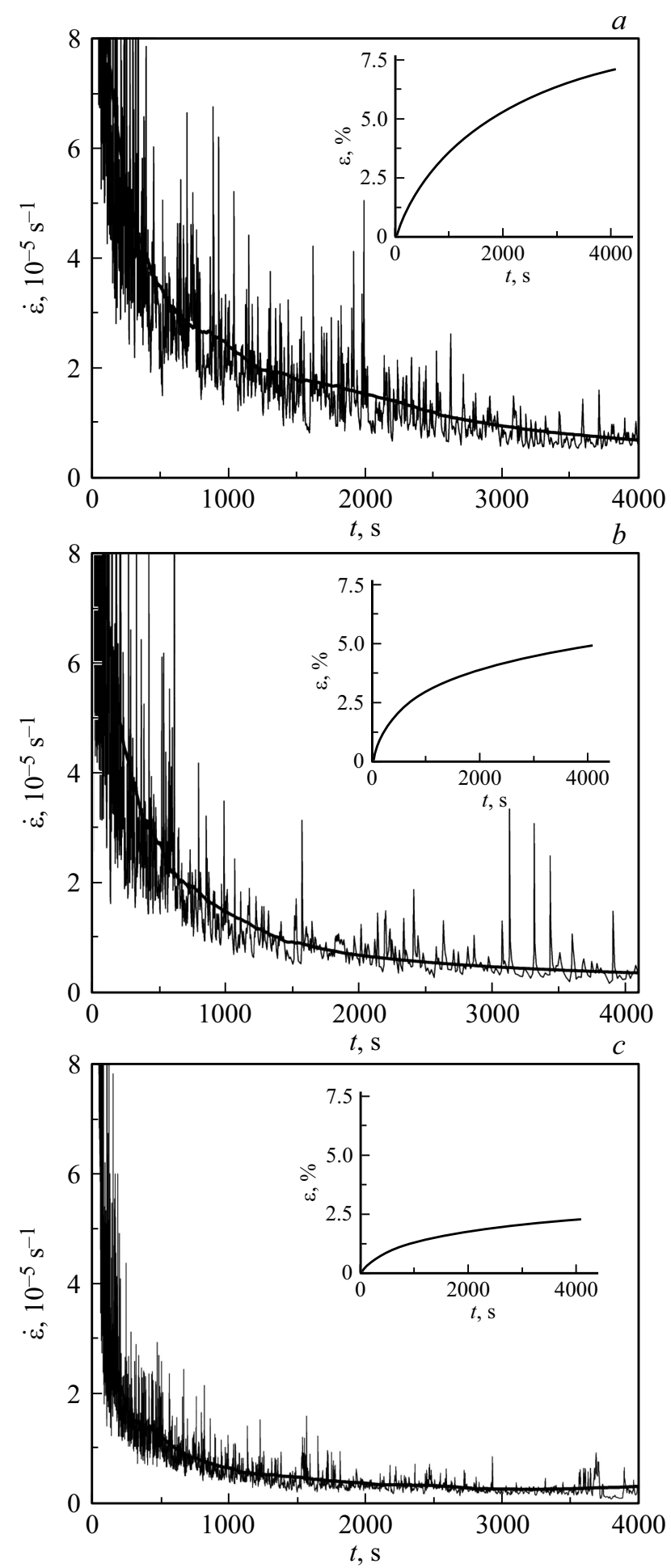

Рис. 1. Экспериментальные и сглаженные зависимости скорости деформации от времени при сжатии с постоянными напряжениями для UHMV PE $(a)$, композитов UHMV PE-1 mass\% Hal $(b)$ и UHMV PE-3 mass\% Hal $(c)$. На вкладках показаны интегральные зависимости величины деформации от времени. 


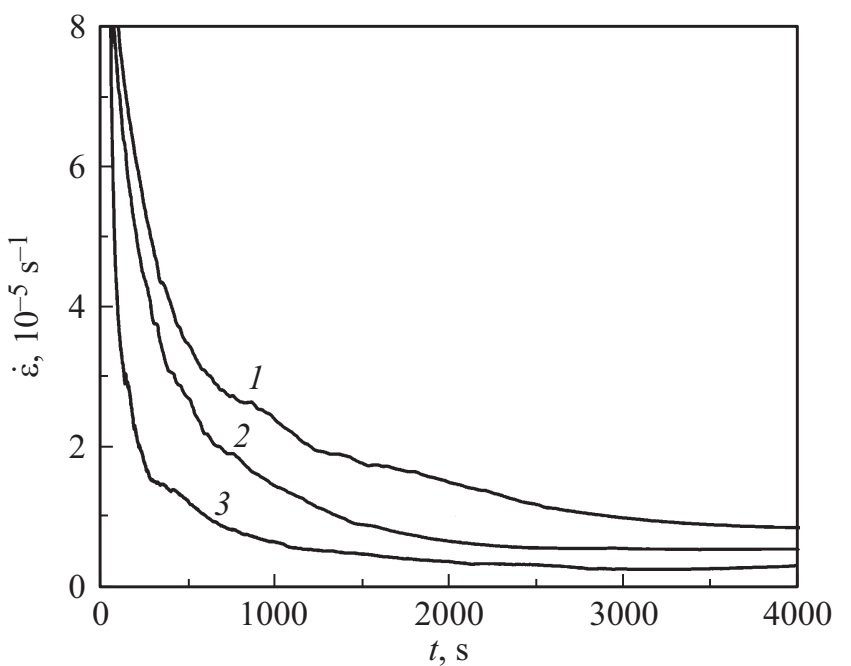

Рис. 2. Сглаженные кривые, перенесенные с рис. $1, a-c$ для UHMV PE (1), композитов UHMV PE-1 mass\% Hal (2) и UHMV PE-3 mass\% Hal (3).

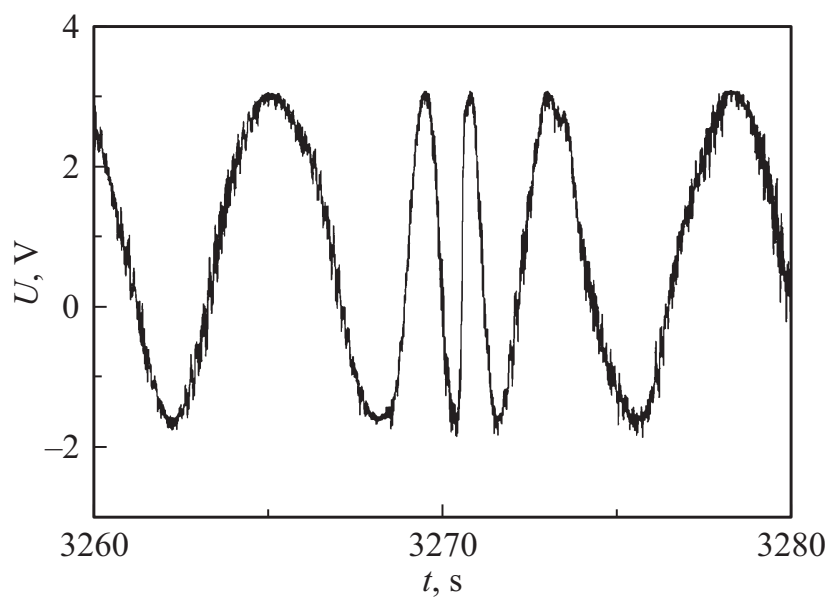

Рис. 3. Пример неравномерности скорости деформации композита UHMV PE-1 mass\% Hal.

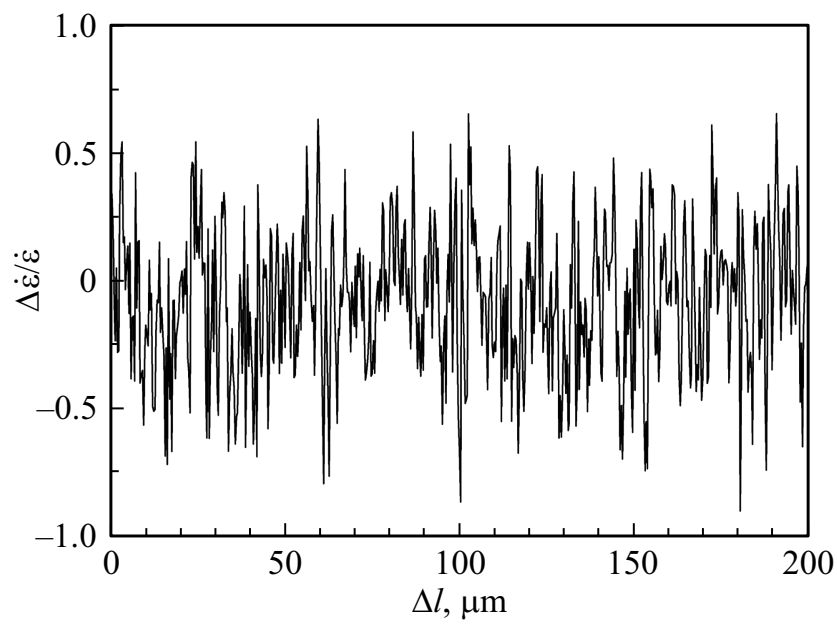

Рис. 4. Зависимость относительной величины скачка скорости деформации $\Delta \dot{\varepsilon} / \dot{\varepsilon}$ от изменения длины образца $\Delta l$ (без учета знака $\Delta l$ ) для композита UHMV PE-3 mass\% Hal.
Таблица 1. Характеристики микро- и нанометровых скачков деформации в UHMV PE и в композитах UHMV PE-( $1-3$ mass\% Hal $)$

\begin{tabular}{|c|c|c|c|c|c|}
\hline $\begin{array}{c}{[\mathrm{Hal}],} \\
\text { mass\% }\end{array}$ & $S_{\overline{\Delta \dot{\varepsilon}}} 10^{-5}, \mathrm{~s}^{-1}$ & $S_{\overline{\Delta \dot{\varepsilon} / \varepsilon}}$ & $L, \mu \mathrm{m}$ & $S_{\overline{\Delta U / U_{0}}}$ & $L_{1}, \mathrm{~nm}$ \\
\hline 0 & 1.616 & 0.327 & $\begin{array}{r}19.6 \pm 6.8 \\
7.5 \pm 1.8 \\
2.9 \pm 0.9\end{array}$ & 0.127 & $\begin{array}{c}>100 \\
16.3 \pm 4.1 \\
2.2 \pm 0.5\end{array}$ \\
\hline 1 & 1.409 & 0.356 & $\begin{array}{r}22.2 \pm 6.5 \\
8.9 \pm 2.2 \\
2.6 \pm 0.9\end{array}$ & 0.149 & $\begin{array}{c}>100 \\
14.1 \pm 3.8 \\
2.4 \pm 0.4\end{array}$ \\
\hline 3 & 0.926 & 0.364 & $\begin{array}{r}22.1 \pm 5.9 \\
8.7 \pm 1.8 \\
2.8 \pm 0.4\end{array}$ & 0.197 & $\begin{aligned} 90.4 & \pm 25.1 \\
11.0 & \pm 1.9 \\
2.3 & \pm 0.2\end{aligned}$ \\
\hline
\end{tabular}

рам $L$ (рис. 5). Диапазон изменения скачков по размерам составлял от 1.5 до $\sim 35 \mu \mathrm{m}$ для всех образцов, что соответствует деформации $0.025-0.6 \%$. Большие скачки были единичными и состояли из скачков меньшего размера. В композите UHMV PE-3 mass\% Hal заметно возрастала доля больших скачков (больших $15 \mu \mathrm{m}$ ), что приводило к уменьшению подвижности и отмеченному выше росту сопротивления деформации. В области малых размеров можно выделить два характерных размера: $2-3$ и 7-9 $\mu \mathrm{m}$. Перераспределение их вкладов в деформацию, возможно, связано с различием в скорости и величине деформации. Подобное явление наблюдалось нами ранее в [14]. Если условно разбить все скачки на 3 группы: до $5 \mu \mathrm{m}$, от 5 до $12 \mu \mathrm{m}$ и все остальные, то получим средние значения величины скачков каждой группы, приведенные в табл. 1. Их изменение с увеличением содержания Hal можно сопоставить с изменением величины среднеквадратичного отклонения скорости деформации $S_{\overline{\Delta \dot{\varepsilon}}}$ и $S_{\overline{\Delta \dot{\varepsilon}(\tilde{\varepsilon}}}$, которые также приведены в табл. 1. Как следует из данных табл. 1, размеры малых микрометровых скачков изменяются слабо, а средние и большие скачки деформации у композита больше, чем у исходного UHMV PE. Подобным образом изменяется величина $S_{\overline{\Delta \bar{\varepsilon} \varepsilon}}$. Заметим, что абсолютная величина скачков скорости деформации $S_{\overline{\Delta \varepsilon}}$ имеет обратную тенденцию (см. табл. 1 и рис. $1, a, b, c$ ), но уменьшение скорости превалирует в данном случае над изменением абсолютной величины ее скачков.

Характеристики нанометровых скачков деформации $S_{\overline{\Delta U / U_{0}}}$ и $L_{1}$, аналогичные по смыслу рассмотренным выше $S_{\overline{\Delta \dot{\varepsilon} / \varepsilon}}$ и $L$ для микрометровых скачков, определялись по искажению формы биений [12]. Для каждого материала произвольным образом выбирали несколько одиночных биений, каждое из которых аппроксимировали зависимостью $U_{\text {cal }}=U_{0} \sin \left(\omega\left(t-t_{0}\right)\right)$, где $U_{0}$ и $\omega-$ амплитуда и частота расчетного сигнала, а $t_{0}-$ время, соответствующее нулевому значению сигнала. Рассчитывали среднеквадратичное отклонение сигнала $S_{\overline{\Delta U / U_{0}}}$ при изменении длины образца $\Delta l_{0}=325 \mathrm{~nm},(\Delta U-$ раз- 

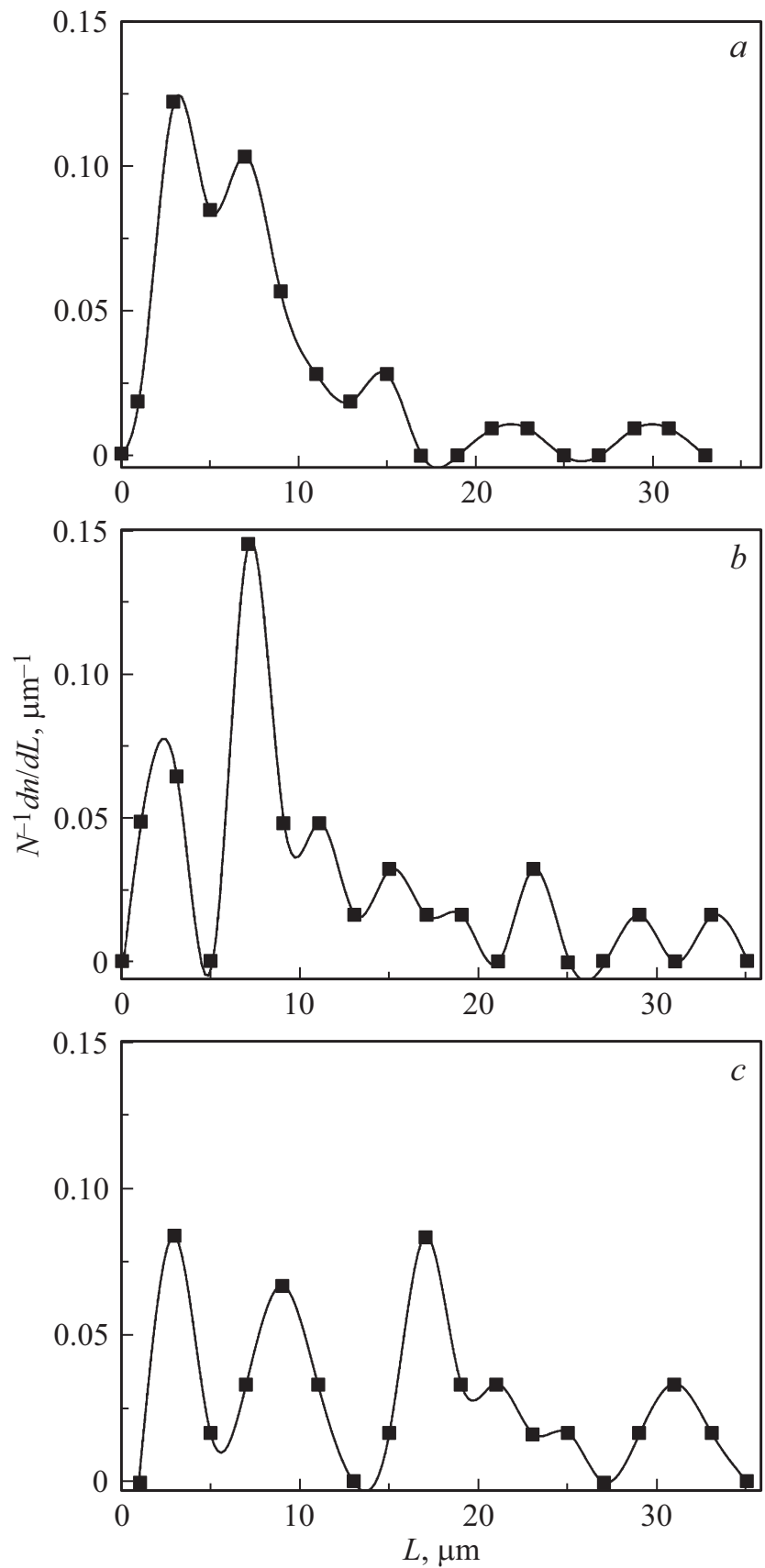

Рис. 5. Распределение микрометровых скачков $L$ по размерам для UHMV PE $(a)$, композитов UHMV PE-1 mass\% Hal (b) и UHMV PE-3 mass\% Hal $(c)$.

ница между расчетной и экспериментальной величиной сигнала), и размер нанометровых скачков деформации $L_{1}$. Примеры зависимостей $\Delta U / U_{0}(\Delta l)$ приведены на рис. $6, a, b$, а полные результаты сведены в табл. 1 .

Как следует из табл. 1, закономерности изменения $S_{\overline{\Delta U / U_{0}}}$ и $L_{1}$ при увеличении концентрации Hal для нанометровых скачков в целом подобны описанным выше для аналогичных характеристик микрометровых скачков: при увеличении концентрации галлуазита $S_{\overline{\Delta U / U_{0}}}$ растет, а размеры больших и малых скачков изменяются слабо.
Единственным отличием является небольшое уменьшение размеров средних по величине $(10-20 \mathrm{~nm})$ скачков у композитов по сравнению с исходным UHMV PE, в то время как микрометровые скачки средних размеров $(5-12 \mu \mathrm{m})$ изменялись противоположным образом. Возможно, это отличие является характеристикой случайно выбранных отдельных биений. Анализ нанометровых скачков дает лишь общее представление об их величине, поскольку измерения проводятся на 2-5 биениях, при этом их общее число может достигать несколько тысяч и более. Естественные шумы также могут оказывать влияние на измерения.

Влияние на величну скачков временных эффектов можно рассчитать по данным рис. 6. На рис. 6, $a$ показано одно полное биение $(t \approx 5 s)$, состоящее из 25 скачков с $L_{1} \approx 12 \mathrm{~nm}$. Следовательно, среднее время одного скачка составляет $\sim 200 \mathrm{~ms}$. Длительность самых малых скачков (рис. $6, b$ ) можно приближенно оценить в $40 \mathrm{~ms}$ (10 скачков на 1/12 одного биения). Поэтому при выбранных условиях временные эффекты в соответствии со сделанными выше предположениями не сказываются на величине нанометровых скачков. Однако если ско-
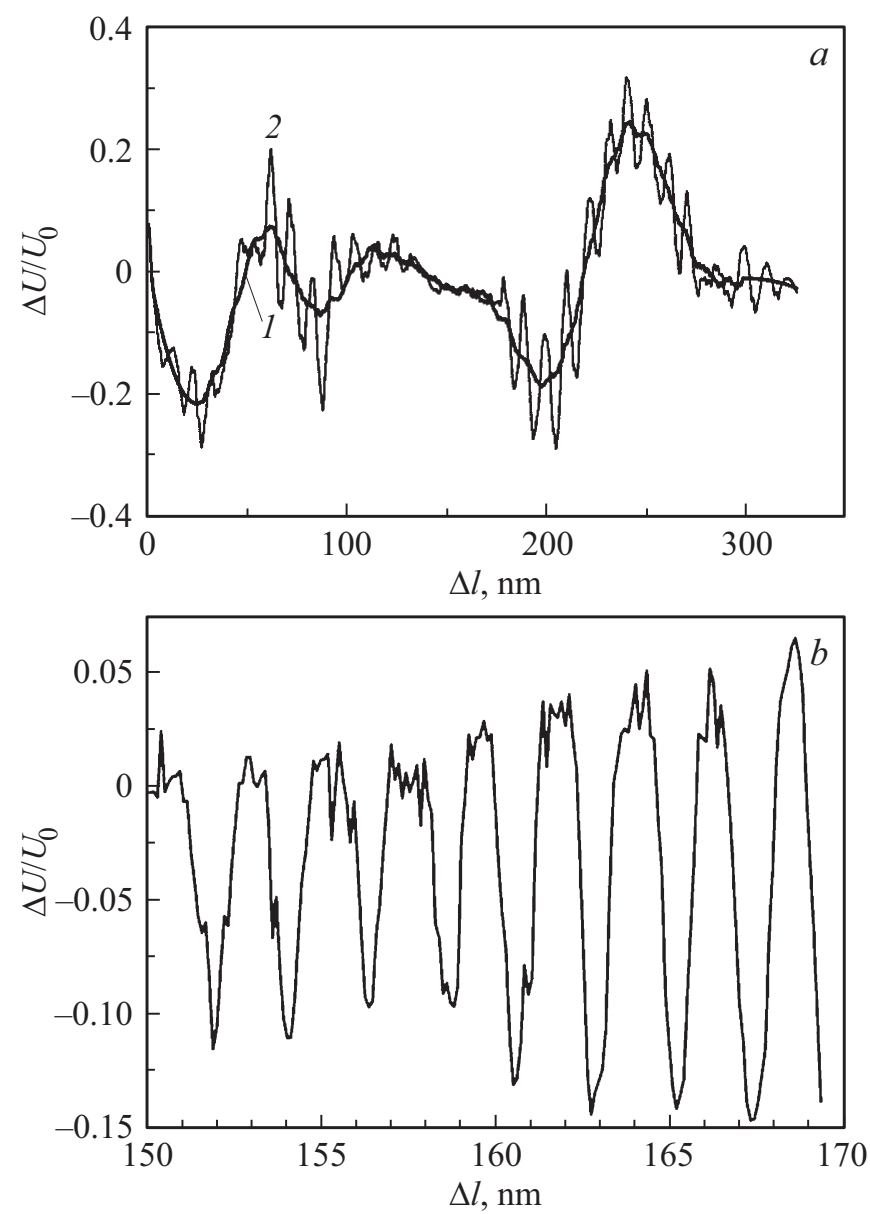

Рис. 6. Фрагменты интерферограммы с нанометровыми скачками деформации $L_{1}:(a)$ большого $(\sim 80 \mathrm{~nm})$ и среднего размера $(\sim 12 \mathrm{~nm}),(b)$ малого размера $(\sim 2.4 \mathrm{~nm})$ для композита UHMV PE-3 mass\% Hal. 
рость деформации увеличить, например в 10 раз, то их следует учитывать.

Помимо исследования малых деформаций было проведено измерение теплового эффекта при плавлении UHMV PE и композитов UHMV PE-(1-3 mass\% Hal $)$ методом дифференциальной сканирующей калориметрии (ДСК) и предпринята попытка связать размеры нанометровых скачков деформации с элементами структуры полимера.

Величина скачков деформации может отражать наличие в структуре полимера некоторых устойчивых для данной области деформаций образований, перемещение которых под действием силы обеспечивает его деформацию. Известно, что для малых частиц неорганических материалов наблюдается фазовый размерный эффект, т е. зависимость теплофизических характеристик от размера частиц [15-17].

Отличия в фазовом состоянии малых частиц обусловлены различными внутренними причинами, между которыми существует определенная взаимосвязь. Размерный эффект выражается, в частности, в смещении температур фазовых переходов, в том числе, плавления. Это смещение по температуре $\Delta T$ зависит от нескольких фаторов и в общем виде определяется выражением $\Delta T / T_{0}=\gamma K / \Delta H \rho R$, где $\gamma-$ поверхностная энергия, $\rho$ - плотность кристалла, $\Delta H$ - энтальпия перехода, $R$ - характерный размер малой частицы или толщина тонкой пленки, $K-$ коэффициент формы, $\Delta T=T_{0}-T_{m}$, где $T_{0}$ и $T_{m}-$ температуры фазового перехода в массивном материале и малой частицы, соответственно.

Аналогичное соотношение $T_{m}=T_{0}\left(1-2 \gamma_{1} / \Delta H_{0} \rho L_{0}\right)$, называемое уравнением Томсона-Гиббса $[18,19]$, широко используется при исследовании плавления и кристаллизации ламелярных полимерных кристаллов, в частности, полиэтилена. В качестве размерного параметра при этом используется толщина ламели $\left(L_{0}\right)$, торцевая

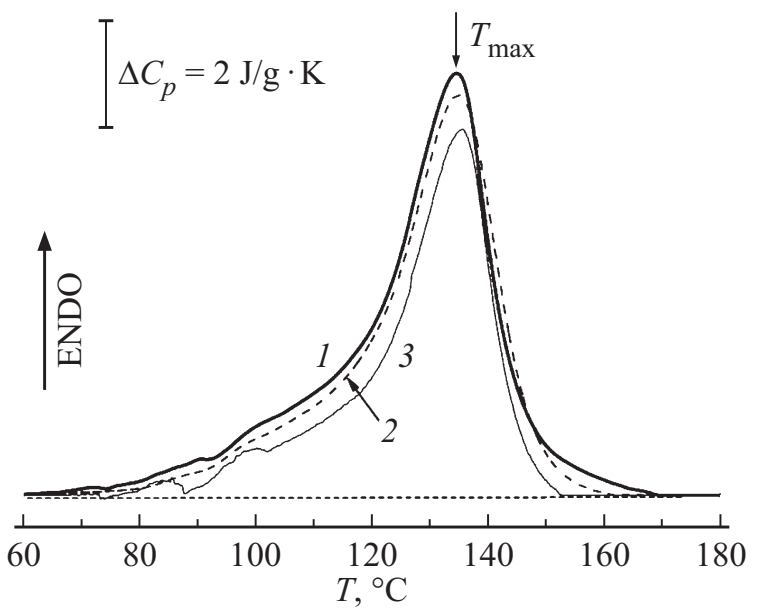

Рис. 7. Кривые ДСК в области плавления для UHMV PE (1), композитов UHMV PE-1 mass\% Hal (2) и UHMV PE-3 mass\% Hal (3).
Таблица 2. Значения температуры и энтальпии плавления, кристалличности в UHMV PE и в композитах UHMV PE-(1-3 mass\% Hal), рассчитанные на основании калориметрических данных

\begin{tabular}{c|c|c|c}
\hline$[\mathrm{Hal}]$, mass $\%$ & $T_{\max },{ }^{\circ} \mathrm{C}$ & $\Delta H, \mathrm{~J} / \mathrm{g}$ & $\chi, \%$ \\
\hline 0 & 134.4 & 214 & 74 \\
1 & 134.8 & 198 & 68 \\
3 & 135.4 & 156 & 54
\end{tabular}

поверхностная энергия $(\gamma)$, энтальпия $\Delta H_{0}$ и температура плавления $T_{0}$ равновесного бесконечного кристалла. Для полиэтилена $\Delta H_{0}=290 \mathrm{~J} / \mathrm{g}, \gamma=87.4 \cdot 10^{-3} \mathrm{~J} / \mathrm{m}^{2}$, $T_{0}=414.5 \mathrm{~K}$ [20]. При кристаллизации ПЭ и ПЭ с добавками образуется структура, состоящая из ламелей, имеющих кристаллический сердечник, и аморфных межламелярных прослоек, располагающихся вдоль торцевых и боковых поверхностей ламелей. Объем ламелей и, соответственно, степень кристалличности при кристаллизации зависит от нескольких параметров, один из которых - толщину ламелей $L_{0}$ можно оценить по приведенному выше соотношению ТомсонаГиббса.

На рис. 7 представлены кривые ДСК, полученные при нагревании закристаллизованных образцов ПЭ и композита ПЭ-галлуазит. На кривых видны эндотермические пики плавления, по которым были получены температуры плавления $T_{\max }$ и рассчитаны степени кристалличности $\chi$ по соотношению $\chi=\left(\Delta H / \Delta H_{0}\right)$, где $\Delta H$ - энтальпия плавления (величина, пропорциональная площади пика), $\Delta H_{0}=290 \mathrm{~J} / \mathrm{g}$. Полученные значения параметров пика плавления представлены в табл. 2.

Из таблицы видно, что в композитах происходит уменьшение энергии плавления $\Delta H$ и, соответственно, степени кристалличности, а температура плавления $T_{\max }$ СВМПЭ и композита изменяется незначительно. Оценка толщины ламелей по уравнению Томсона-Гиббса дает значение $L_{0}=15 \pm 3 \mathrm{~nm}$, сопоставимое с размером деформационных скачков нанометрового уровня в диапазоне $10-20 \mathrm{~nm}$.

\section{4. Заключение}

Работы последних лет [10-14], посвященные исследованию скачков деформации, показали, что в совершенно различных по своей природе твердых телах (полимерах, металлах, композитах) существует несколько уровней деформации, которым соответствуют скачки ее скорости разной величины. Величина скачков отражает состояние структуры материала, которое определяется его предысторией и характером внешнего воздействия. В настоящей работе рассмотрено влияние небольших добавок Hal в UHMV PE на скорость деформации и характеристики скачков скорости и величины деформации. Обнаруженные скачки деформации имели 
размеры от $\sim 2 \mathrm{~nm}$ до $\sim 20 \mu \mathrm{m}$. Уменьшение скорости деформации с ростом содержания Hal проявилось в увеличении среднеквадратичного отклонения скорости на начальном участке деформации, как для скачков микро-, так и нанометрового уровня. Величина скачков при этом изменялась слабо, заметное изменение их величины было обнаружено лишь для скачков с размерами $7-9 \mu \mathrm{m}$ и $10-20 \mathrm{~nm}$. Размеры последних близки к толщине ламелей, определенной методом ДСК. Калориметрические измерения показали, что температура плавления, при добавлении в UHMV PE 1-3 mass\% Hal практически не изменяется, а энергия перехода и степень кристалличности уменьшаются, что можно связать с ограничением подвижности боковых поверхностей ламелей при кристаллизации под влиянием взаимодействия с нано- и микрочастицами галлуазита. Об этом может свидетельствовать обнаруженное нами увеличение доли больших скачков при деформации композитов UHMV PE-(1-3 mass\% Hal), что приводит к уменьшению подвижности и росту сопротивления деформации.

\section{Список литературы}

[1] Y. Lvov, E. Abdullayev. Prog. Polym. Sci. 38, 1690 (2013).

[2] P. Yuan, D. Tan, F. Annabi-Bergaya. Appl. Clay Sci. 112-113, 75 (2015).

[3] M. Liu, Z. Jia, D. Jia, C. Zhou. Prog. Polym. Sci. 39, 1498 (2014).

[4] Y. Lvov, W. Wang, L. Zhang, R. Fakhrullin. Adv. Mater. 28, 1227 (2016).

[5] U.F. Handge, K. Hedicke-Hochstotter, V. Altstadt. Polymer 51, 2690 (2010).

[6] M. Du, B. Guo, Y. Lei, M. Liu, D. Jia. Polymer 49, 4871 (2008).

[7] V.P. Singh, K.K. Vimal, G.S. Kapur, S. Sharma, V. Choudhary. J. Polym. Res. 23, 1 (2016).

[8] M. Du, B. Guo, J. Wan, Q. Zou, D. Jia. J. Polym. Res. 17, 109 (2010).

[9] Н.Н. Песчанская. Высокомолекуляр. соединения А 31, 1181 (1989).

[10] В.В. Шпейзман, Н.Н. Песчанская, Б.И. Смирнов. ФТТ 50, 815 (2008).

[11] В.В. Шпейзман, Н.Н. Песчанская, Т.С. Орлова, Б.И. Смирнов. ФТТ 51, 2315 (2009).

[12] В.В. Шпейзман, Н.Н. Песчанская. ФТТ 53, 1169 (2011).

[13] Н.Н. Песчанская, П.Н. Якушев, В.В. Шпейзман, А.С. Смолянский, А.С. Шведов, В.Г. Черемисов. ФТТ 52, 1837 (2010).

[14] В.В. Шпейзман, П.Н. Якушев, Ж.В. Мухина, Е.В. Кузнецов, .С. Смолянский. ФТТ 55, 923 (2013).

[15] Н.Т. Гладких, С.В. Дукаров, А.П. Крышталь, В.И. Ларин, В.Н. Сухов, С.И. Богатыренко. Поверхностные явления и фазовые превращения в конденсированных пленках. ХНУ им. В.Н. Каразина, Харьков. (2004). 276 с.

[16] А.И. Гусев. Наноматериалы, наноструктуры, нанотехнологии. Наука, М. (2007). 416 с.
[17] A.I. Gusev, A.A. Rempel. Nanocrystalline Materials. Int. Sci. Publ., (2004). 351 p.

[18] J.I. Lauritzen, J.D. Hoffman. J. Res. Nature Stand. A 64, 73 (1960).

[19] J.D. Hoffman, J.J. Weeks. J. Res. Nature Stand. A 66, 13 (1962).

[20] Б. Вундерлих. Физика макромолекул. Мир, М. (1984). Т. 3. $484 \mathrm{c}$.

Редактор Т.В. Василевская 